\title{
Development of solid paediatric oral dosage forms
}

\author{
Egide Kayitare \\ Supervisors: Jean Paul Remon, Chris Vervaet \\ Department of Pharmaceutics, Ghent University
}

Public defence: 26 April 2010

The paediatric population includes a category of patients with dynamic changes (due to the continuous body growth). Therefore their treatment requires individualized drug dosing (according to body weight) and the availability of different dosage forms for the same treatment due to different ability of paediatric patients to handle liquid (too much for older children and adolescents) or solid dosage forms (so hard and too big to be swallowed by infants and small children). Mostly, liquid and solid dosage forms are necessary to satisfy the needs for all age-range of paediatrics. However, on the market, there is a lack of appropriate dosage forms for paediatric patients.

Chapter I describes the different problems resulting in the lack of paediatric drug dosage forms on the market and the off-label use of adult drug dosage forms in paediatrics. This chapter gives an overview of the available oral drug dosage forms during childhood and the issues related to oral liquid dosage forms.

The objective of this study was to develop paediatric dosage forms which will be suitable for different paediatric age groups and allowing to obtain doses related to body weight or body surface area, taking into account two pandemic diseases in Africa, namely malaria and HIV/AIDS.

To achieve this objective, two formulations (powder for reconstitution and tablets with innovative design) have been developed. Innovative design consisted of tablets breakable in multiple subunits (8) allowing sufficient flexibility in dosing according to child's body weight or body surface area, and fast disintegrating, allowing drug administration as liquid (after dispersion in a small amount of water) or as solid depending on child's ability of swallowing.

Development of dosage forms followed the common steps such as pre-formulation study, formulation and preparation of dosage form, in vitro test and vivo test (bioavailability of drugs in healthy adults before in vivo study in children).

Chapter 2 focused on the development of taste-masked oral quinine dosage forms for treatment of children with uncomplicated malaria. A water quinine insoluble quinine pamoate salt was formed following precipitation of quinine sulphate by disodium pamoate acid. Two quinine pamoate formulations were developed: a powder for reconstitution and a novel tablet for flexible dosing. The reconstituted suspension $(150 \mathrm{mg} / 5 \mathrm{ml})$ was stable at least for I week in ambient conditions. Quinine pamoate powder was also used to formulate fast-disintegrating tablets by using a specific tablet design (rectangular tablet which can be divided into 8 subunits) to allow dosing flexibility. This novel tablet has 
size, shape and specific score lines for breaking accurately and with minimum weight loss. The physical properties of the tablets were evaluated in vitro: quinine pamoate tablets complied with the pharmacopoeial requirements for mass uniformity, friability, content uniformity, breakability, disintegration and fast dissolution.

Since the novel tablets were fast disintegrating, they can be administered to children as liquid (after dispersion in a small amount of water) or as solid, according to child's ability to swallow solid dosage forms.

In chapter 3 , quinine bioavailability of the innovative tablets was investigated in healthy adults $(n=18)$, and the steady state and efficacy were evaluated by using them in treatment of children (aged between 6 and 59 months) with uncomplicated Plasmodium falciparum malaria $\left(n=5^{6}\right)$ via a 7 -day regimen of $8 \mathrm{mg}$ quinine $/ \mathrm{kg}$. The results showed that the quinine pharmacokinetic parameters after single administration of a quinine pamoate tablet were similar to a commercially available quinine sulphate tablet.

Treatment of children suffering from uncomplicated malaria showed a fast decline in parasitaemia $(28.6 \% / 24 \mathrm{~h})$, a reduction rate of fever (all children were apyretic after $72 \mathrm{~h}$ ). The steady state quinine plasma concentration ranged between 5.7 and $15.8 \mu \mathrm{g} / \mathrm{ml}$, proving the efficacy of the quinine pamoate tablets against Plasmodium falciparum.

These results were similar to those obtained in other studies when quinine was administered using a dose of $8 \mathrm{mg}$ quinine $/ \mathrm{kg} / 8$ hours. Novel (fast-dispersible and breakable) taste-masked quinine pamoate tablets provide several advantages over the current dosage forms: improved dosing accuracy (dose adjusted to body weight with interval of $2.5 \mathrm{~kg}$ body weight), easy administration to children of different ages.

In Chapter 4 , the same tablet design was used to develop a novel fixed dose combination (FDC) tablet containing $300 \mathrm{mg}$ zidovudine (AZT) and $\mathrm{r} 60 \mathrm{mg}$ lamivudine ( $3 \mathrm{TC}$ ) according to the recommendation of WHO. The results of a breakability test performed by nurses showed that the novel FDC tablets were easily broken along the score lines into pieces having a reproducible weight $(\mathrm{I} 25 . \mathrm{I} \pm 5 \mathrm{mg}$, R.S.D. $=4.4 \%$, with limited weight loss $(0.3 \%)$, independent on their size or the user.

The results of disintegration test showed that the novel FDC tablets as well as its subunits disintegrated fast ( $<20 \mathrm{~s}$ ), allowing easy administration of tablet or subunits to children either after dispersion in a liquid or as small tablets. Within 30 min dissolution, more than $95 \%$ zidovudine and lamivudine was released from the novel tablets. The dissolution profile of novel tablets was similar to those obtained with tablets commercially available "Duovir(?), the similarity factors $\mathrm{f}_{2}$ were above 50 for both AZT and 3 TC.

Chapter 5 describes a crossover bioavailability study. The oral bioavailability of zidovudine and lamivudine administered as the novel FDC tablets and as the commercially available Duovir ${ }^{\circledR}$ tablets was evaluated in $\mathrm{I} 8$ healthy adult volunteers. The results showed that there was no significant difference in pharmacokinetic parameters (Cmax, tmax and AUCo-12 h values) between Duovir and the novel paediatric tablets ( $t$-test, $\mathrm{p}>0.005$ ). The relative bioavailability of zidovudine and lamivudine was $101.8 \%$ and $101.4 \%$ respectively, considering Duovir ${ }^{\circledR}$ tablets as reference formulation. In this chapter, the steady state concentration of both zidovudine and lamivudine was assessed in 36 children with 
HIV. This study was performed in two groups of 18 children with HIV: a control group which continued their usual dosage combination of zidovudine and lamivudine and a trial group who shifted from their usual FDC tablets (Duovir ${ }^{\circledR}$ or Duovir ${ }^{\circledR}$ ) to the experimental FDC tablets for Io days. No significant differences were observed between the pharmacokinetics of zidovudine and lamivudine in the control and rial group. The relative bioavailability of zidovudine and lamivudine was $93.5 \%$ and $97.3 \%$, respectively. Chapter 6 includes general conclusions and future perspectives. 